\title{
Beribéri Pós Bypass Gástrico: Uma Complicação Não Tão Rara. Relato de Dois Casos e Revisão da Literatura
}

\author{
Lilian F.A. Alves \\ Ricardo M. Gonçalves \\ Giovana V. Cordeiro \\ Márcio W. Lauria \\ Adauto V. Ramos
}

Serviço de Endocrinologia do Hospital Felicio Rocho, Belo Horizonte, $M G$.
Recebido em 03/10/05

Revisado em 06/02/06

Aceito em 12/02/06

\section{RESUMO}

É crescente o número de pacientes submetidos à cirurgia bariátrica para tratamento da obesidade mórbida; devido a isso, estão reaparecendo deficiências nutricionais com as quais muitos médicos já não estão familiarizados. No pós-operatório, podem ocorrer vários distúrbios nutricionais, sendo um deles a deficiência de tiamina (beribéri). A deficiência de tiamina e/ou vitamina B12 pode corresponder a $40 \%$ dos casos de neuropatia após a cirurgia bariátrica. Serão descritos dois pacientes com a clínica de neuropatia periférica e síndrome de Wernicke-Korsakoff. Alguns meses após a cirurgia, eles apresentaram prostração, depressão, confusão mental e nistagmo, associados a dor e parestesia dos membros (principalmente inferiores). Com a hipótese diagnóstica de beribéri, foi iniciado o tratamento com tiamina. Um dos pacientes apresentou melhora completa do quadro neurológico, porém o outro permaneceu com déficit motor, sendo aquele em que decorreu o maior tempo entre o aparecimento dos sintomas e o início do tratamento. Estes casos servem para nos alertar sobre a importância da vigilância nutricional após a cirurgia bariátrica. (Arq Bras Endocrinol Metab 2006;50/3:564-568)

Descritores: Cirurgia bariátrica; Bypass gástrico; Distúrbios nutricionais; Beribéri

\begin{abstract}
Beriberi After Bariatric Surgery: Not An Unusual Complication. Report of Two Cases and Literature Review.

The number of patients submitted to bariatric surgery to treat morbid obesity is increasing, therefore, some nutritional deficiencies, with which many physicians are no longer familiarized, are reappearing. Postoperatively, many nutritional disorders may occur, one of them is thiamine deficiency (beriberi). The thiamine and/or vitamin B12 deficiency can correspond to $40 \%$ of the neuropathy cases after bariatric surgery. Two patients with the clinic of peripheral neuropathy and Wernicke-Korsakoff syndrome will be reported. Some months after the surgery, they presented prostration, depression, mental confusion and nystagmus, associated with pain and paresthesia in limbs (especially lower limbs). With the diagnostic hypothesis of beriberi, the treatment with thiamine started. One of the patients presented complete improvement of the neurological symptoms, however the other one remained with motor deficiency, exactly the one who spent a longer period of time between the symptoms appearance and the treatment beginning. These cases serve to alert us about the importance of nutritional vigilance after bariatric surgery. (Arq Bras Endocrinol Metab 2006;50/3:564-568)
\end{abstract}

Keywords: Bariatric surgery; Gastric bypass; Nutrient deficiencies; Beriberi 
A PREVALÊNCIA DA OBESIDADE VEM aumentando no Brasil e em todo o mundo. No Brasil, dados do Instituto Brasileiro de Geografia e Estatística (IBGE) de 2002-2003 mostraram uma prevalência de sobrepeso e obesidade de $40 \%$ na população adulta, sendo que $8,9 \%$ dos homens e $13,1 \%$ das mulheres tinham obesidade. Nos Estados Unidos, 60\% dos homens e 55\% das mulheres tinham sobrepeso e/ou obesidade em 1994 (dados do National Health and Nutrition Examination Survey III - NHANES III). Também na adolescência, a obesidade tornou-se uma doença comum, sendo que 4,1 milhões de adolescentes americanos têm índice de massa corporal (IMC) acima do percentil 95 (1).

A cirurgia bariátrica vem sendo cada vez mais usada para o tratamento da obesidade mórbida em adultos e adolescentes, mostrando-se um procedimento seguro (1). No bypass gástrico, a taxa de distúrbios nutricionais e eletrolíticos é de $17 \%$ e a mortalidade varia entre $0,3-1,9 \%(2,3)$. O tratamento cirúrgico está indicado em pacientes com IMC maior que 40 $\mathrm{kg} / \mathrm{m}^{2}$ ou maior que $35 \mathrm{~kg} / \mathrm{m}^{2}$ quando associado a comorbidades. Ele proporciona uma redução de peso sustentada, podendo ocorrer perda de $30-40 \%$ do peso inicial, além de ocorrer melhora da qualidade de vida e da maioria das comorbidades associadas, como diabetes tipo 2 , hipertensão arterial sistêmica e disfunção cardiovascular (4).

A cirurgia bariátrica pode ser exclusivamente restritiva (ex. banda gástrica), exclusivamente disabsortiva (ex. derivação biliopancreática) ou restritivodisabsortiva (ex. bypass gástrico em Y de Roux) (5). Esta última é uma cirurgia que promove saciedade precoce em decorrência da redução gástrica e má absorção em virtude do pequeno espaço intestinal em que se misturam os alimentos aos sucos digestivos (canal comum). O grau de má absorção está relacionado à extensão do canal comum (4).

Os distúrbios nutricionais mais freqüentes após a cirurgia bariátrica são: a desnutrição protéica, as deficiências de ferro e zinco e as deficiências vitamínicas (tiamina, niacina, cobalamina, ácido fólico, vitamina $\mathrm{D}$, vitamina A e vitamina $\mathrm{E}$ ) (6-8). As deficiências parecem ser mais significativas após procedimentos disabsortivos, mas também ocorrem nos restritivos (8). Os possíveis mecanismos são: a ingestão nutricional deficiente, a má absorção decorrente da técnica cirúrgica e a pobre aderência à reposição de polivitamínicos.

Abaixo, descreveremos dois casos de pacientes com obesidade grau III que evoluíram com beribéri alguns meses após a realização do bypass gástrico.

\section{RELATO DOS CASOS}

\section{Caso 1}

Paciente masculino de 5l anos, com IMC de 44 $\mathrm{kg} / \mathrm{m}^{2}$, submetido ao bypass gástrico em Y de Roux. No pós-operatório imediato, teve como complicações estenose do Y de Roux, infecção com deiscência da ferida operatória e trombose venosa profunda. Cinco meses após a cirurgia, procurou atendimento hospitalar com queixas de sonolência, confusão mental e fraqueza, associados a dor e perda da movimentação dos membros (principalmente inferiores) iniciados há 6 dias, além de quadro depressivo. Havia perdido 52 $\mathrm{kg}$ desde a cirurgia. No exame físico, apresentava torpor, nistagmo horizontal e tetraparesia de membros. Os exames laboratoriais mostraram funções renal e hepática, ionograma e hemograma normais e hipoalbuminemia (tabela 1). Realizaram-se endoscopia digestiva alta, ultra-sonografia abdominal e tomografia computadorizada de crânio, que estavam normais. Após a suspeita clínica de beribéri, foi iniciada a reposição venosa de tiamina $(100 \mathrm{mg} /$ dia $)$ e, após 10 dias, por via oral $(600 \mathrm{mg} / \mathrm{dia})$.

Teve melhora total da confusão mental e parcial da paresia e dor em membros. No momento da alta, conseguia fazer movimentação ativa dos membros, porém apresentava hipotonia e não tolerava o ortostatismo. Não seguiu as orientações de realizar acompanhamento médico e fisioterápico e permaneceu com paraparesia, sem conseguir deambular.

\section{Caso 2}

Paciente feminina de 19 anos, com IMC de 47 $\mathrm{kg} / \mathrm{m}^{2}$, procurou atendimento hospitalar no quarto mês após o bypass gástrico em Y de Roux com queixas de hiporexia, diminuição da acuidade visual, disacusia, disartria, além de paresia, parestesia e dor em membros superiores e inferiores, sendo pior nestes. Referia vômitos, hiporexia e depressão iniciados há 30 dias. Encontrava-se consciente, porém com déficit de memória e distúrbio de comportamento. O exame físico revelou nistagmo horizontal, tetraparesia flácida e intensa dor ao mínimo toque de membros. Os exames laboratoriais mostraram ácido fólico, vitamina B12, função renal, ionograma, proteínas e hemograma normais (tabela 1). As transaminases hepáticas estavam elevadas, porém com propedêutica para elevação de transaminases sem alterações. Realizaram-se endoscopia digestiva alta, exame contrastado de esôfago-estômago-duodeno, ultra-sonografia abdominal e tomografia de crânio, que estavam normais. 
Tabela 1. Comparação dos exames laboratoriais.

\begin{tabular}{lcc}
\hline Exame (valor de referência) & Caso 1 & Caso 2 \\
\hline TGO (12-46 U/L) & 24 & 253 \\
TGP (03-50 U/L) & 41 & 624 \\
FA (50-250 U/L) & 119 & 101 \\
GGT (10-50 U/L) & 64 & 189 \\
Proteína total (6,4-8,3 g/dL) & 5,0 & 5,8 \\
Albumina (3,5-5,5 g/dL) & 2,4 & 3,7 \\
Bb total (até 1,2 mg/dL) & 0,8 & 1,3 \\
Bb direta (até 0,4 mg/dL) & 0,3 & 0,8 \\
Creatinina (0,7-1,2 mg/dL) & 0,7 & 0,8 \\
Hemoglobina $(12-16 \mathrm{~g} \%)$ & 13,1 & 14,1 \\
VCM (80-100 fl) & 93 & 85 \\
Ácido fólico (3-17 ng/mL) & - & 4 \\
Vit B12 (200-950 pg/mL) & 355 & 586 \\
Cálcio total (8,5-10,4 mg/dL) & 7,8 & 9,9 \\
\hline
\end{tabular}

$\mathrm{TGO}=$ transaminase oxalacética; $\mathrm{TGP}=$ transaminase pirúvica: $F A=$ fosfatase alcalina; $G G T=$ gama glutamil transferase; $\mathrm{Bb}$ total= bilirrubina total; $\mathrm{VCM}=$ volume corpuscular médio; vit $\mathrm{B} 12=$ vitamina $\mathrm{B} 12$

Feita a hipótese diagnóstica de beribéri e iniciada a reposição de tiamina. Evoluiu com melhora progressiva dos sintomas apresentados à admissão. Recebeu alta hospitalar sem déficit motor em membros superiores, tolerando ortostatismo e com movimentação ativa em leito. Ainda apresentava discreta dor em membros, porém sem necessidade de analgésicos. Continuou realizando acompanhamento médico e fisioterápico. Atualmente, consegue deambular e as transaminases já se normalizaram.

\section{DISCUSSÃO}

A neuropatia periférica é uma das manifestações clínicas dos distúrbios nutricionais. Um estudo retrospectivo encontrou uma prevalência de $16 \%$ de neuropatia periférica em pacientes submetidos à cirurgia bariátrica (9). Os fatores de risco foram: a quantidade de perda de peso, a persistência de sintomas gástricos, a não aderência ao acompanhamento nutricional, a redução da albumina e transferrina, a presença de complicações pós-operatórias e a presença de bypass jejunoileal (9). A perda rápida de peso foi associada à baixa concentração sérica de vitamina B12, tiamina e ácido fólico. Em alguns pacientes, a neuropatia pode ser atribuída diretamente à deficiência de tiamina ou niacina (9). Os dois casos descritos sugerem beribéri, tendo a neuropatia periférica como uma das manifestações clínicas.

O beribéri é a deficiência da vitamina Bl, também denominada tiamina. Muitos casos registrados estão relacionados ao paciente com vômitos prolongados, inanição e, mais comumente, alcoolismo. Recen- temente, casos de beribéri vêm sendo descritos como uma complicação da cirurgia bariátrica, aparecendo de semanas a meses após a cirurgia, mais comumente em pacientes com vômitos prolongados (9-12).

A tiamina é absorvida no intestino delgado, com absorção máxima no jejuno e íleo $(11,12)$. Possui uma meia vida plasmática de 10-20 dias. A dose diária recomendada em adultos é de $0,5 \mathrm{mg}$ para cada 1000 Kcal ingeridas (13). Os estoques duram de 3-6 semanas em adultos com ingestão oral isocalórica (10). É encontrada em fermento, legumes, carne de porco, aves, cereais de grão inteiro e nozes. O polimento do arroz integral para produzir arroz branco retira o farelo rico em tiamina $(13,14)$. Ela é instável em luz e calor.

O pirofosfato de tiamina, forma ativa da vitamina $\mathrm{Bl}$, é utilizado no metabolismo de carboidratos como catalisador da conversão de piruvato em acetilcoenzima $\mathrm{A}$, uma reação de decarboxilação mediada pela piruvato desidrogenase. Seus níveis séricos podem reduzir após a ingestão oral ou infusão venosa de glicose. A tiamina também tem a função de iniciar a propagação do impulso nervoso, que é independente de sua função de coenzima $(11,15)$.

O beribéri pode ter manifestações neurológicas (centrais ou periféricas) e cardiovasculares. As manifestações iniciais são fadiga, irritabilidade, perda de memória, dor precordial, anorexia, desconforto abdominal e constipação.

O beribéri úmido ocorre na deficiência de tiamina com predomínio de doença cardiovascular. Geralmente se manifesta no paciente com ingestão calórica elevada e atividade física extenuante. Suas principais manifestações são cardiomegalia, cardiomiopatia, insuficiência cardíaca congestiva, edema periférico e taquicardia $(12,16)$.

O beribéri seco é a síndrome de alterações neurológicas periféricas. É mais comum em pacientes com restrição calórica e inatividade relativa. É caracterizado por alterações sensitivas e motoras, que são bilaterais e simétricas. Geralmente se manifesta com dor, parestesia e perda dos reflexos. Começa com parestesias nos artelhos, queimação nos pés, câimbras nas panturrilhas e dor nas pernas. A continuidade da deficiência leva à perda da sensação vibratória e posicional dos dedos, à atrofia da panturrilha e ao pé caído. Os membros inferiores são mais afetados do que os membros superiores (15).

No sistema nervoso central, ocorre a Síndrome de Wernicke-Korsakoff. A psicose de Korsakoff é a alteração da memória recente, confabulação e redução do aprendizado. A encefalopatia de Wernicke é uma tríade de nistagmo com progressão para olftalmoplegia, ataxia e confusão mental que, se não tratada, pode evoluir para coma e morte $(11,16)$. 
Os pacientes descritos apresentaram sinais e sintomas sugestivos de beribéri seco com parestesia e dor em membros, e de Síndrome de Wernicke-Korsakoff com nistagmo, confabulação e confusão mental.

O diagnóstico diferencial deve ser feito com lupus eritematoso sistêmico e outras doenças do tecido conjuntivo, distrofia simpática reflexa, fibromialgia, distúrbio de ansiedade, distúrbio de conversão e neuropatia periférica secundária à deficiência nutricional ou à esclerose múltipla (10).

O diagnóstico laboratorial pode ser realizado através da medida da tiamina sérica, da excreção urinária de tiamina e da transcetolase de eritrócitos (14). Esta última é um dos testes mais confiáveis. Qualquer aumento na atividade enzimática pela administração de pirofosfato de tiamina (PPT) é chamado de efeito PPT. Uma transcetolase eritrocitária baixa e uma resposta maior que $16 \%$ ao PPT indicam provável deficiência de tiamina. Em muitos casos, entretanto, a resposta clínica ao tratamento empírico com tiamina é usada para suportar o diagnóstico de deficiência de tiamina (16).

O tratamento deve ser feito com a administração de 50-200 mg de tiamina parenteral por aproximadamente 7-14 dias e, depois, por via oral até a recuperação completa ser alcançada (10). Metade dos pacientes apresenta melhora completa com o tratamento e metade apresenta apenas melhora parcial ou não tem benefício (16). Nos pacientes descritos, a reposição de tiamina venosa foi feita por 10 dias no primeiro paciente e por 7 dias no segundo. $\mathrm{O}$ primeiro permaneceu com deficiência motora, coincidindo com o maior tempo entre o aparecimento dos sintomas e o início do tratamento.

Chang e cols. (17) encontraram 99 casos de neuropatia aguda após a cirurgia de redução gástrica de 168.010 pacientes operados $(5,9 / 1000)$, sendo que 40 apresentavam deficiência de tiamina e/ou vitamina B12. Ocorreu melhora em 9 de 17 casos de deficiência de vitamina B12, 12 de 29 casos de deficiência de tiamina e 3 de 6 casos com ambas as deficiências.

Vários são os benefícios da redução sustentada de peso através da cirurgia bariátrica. Entretanto, os médicos devem estar atentos para as possíveis deficiências nutricionais associadas à perda de peso, sendo de fundamental importância a realização precoce do diagnóstico. Muitos pacientes submetidos à cirurgia bariátrica terão alguma deficiência nutricional, justificando a suplementação com polivitamínicos em todos os pacientes. Deve ser dada atenção à aderência do paciente ao uso das vitaminas prescritas. $\mathrm{O}$ atraso no diagnóstico pode trazer grande aumento na morbidade e até seqüelas irreparáveis.

\section{REFERÊNCIAS}

1. Barnett SJ, Stanley C, Hanlon M, Acton R, Saltzman DA, Ikramuddin S, et al. Long-term follow-up and the role of surgery in adolescents with morbid obesity. Surg Obes Rel Dis 2005; 1:394-8.

2. Snow V, Barry P, Fitterman N, Qaseem A, Weiss K. Pharmacologic and surgical management of obesity in primary care: a clinical practice guideline from the American College of Physicians. Ann Intern Med 2005; 142:525-31.

3. Maggard MA, Shugarman LR, Suttorp M, Maglione $M$, Sugerman HJ, Livingston EH, et al. Meta-analysis: surgical treatment of obesity. Ann Intern Med 2005; 142:547-59.

4. Choban PS, Jackson B, Poplawski S, Bistolarides P. Bariatric surgery for morbid obesity: why, who, when, how, where, and then what? Cleve Clin J Med 2002;69:897-903.

5. Schneider BE, Mun EC. Surgical management of morbid obesity. Diabetes Care 2005;28:475-80.

6. Fujioka K. Follow-up of nutritional and metabolic problems after bariatric surgery. Diabetes Care 2005;28:481-4.

7. Alvarez-Leite Jl. Nutrient deficiencies secondary to bariatric surgery. Curr Opin Nutr Metab Care 2004; 7:569-75.

8. Bloomberg RD, Fleishman A, Nalle JE, Herron DM, Kini S. Nutritional deficiencies following bariatric surgery: what have we learned? Obes Surg 2005; 15: 145-54.

9. Thaisetthawatkul $P$, Collazo-Clavell ML, Sarr MG, Norell JE, Dyck PJ. A controlled study of peripheral neuropathy after bariatric surgery. Neurology 2004;63:1462-70.

10. Towbin A, Inge TH, Garcia VF, Roehrig HR, Clements $\mathrm{RH}$, Harmon CM, et al. Beriberi after gastric bypass surgery in adolescence. J Pediatr 2004; 145:263-7.

11. Gollobin C, Marcus WY. Bariatric beriberi. Obes Surg 2002; 12:309-11.

12. Nautiyal A, Singh S, Alaimo DJ. Wernicke encephalopathy - an emerging trend after bariatric surgery. Am J Med 2004; 1 17:804-5.

13. Alvarez-Leite Jl. Como abordar as deficiências nutricionais no pós-operatório de cirurgias bariátricas? In: Savassi-Rocha PR, Coelho LGV, Diniz MTC, Nunes TA, editors. Tópicos em gastroenterologia - obesidade e urgências gastroenterológicas. 13a ed. Medsi; 2003. p. 127-40.

14. Cambi MPC, Marchesini JB. Acompanhamento clínico, dieta e medicação. In: Garrido Júnior $A B$, editor. Cirurgia da obesidade. la ed. Atheneu; 2003. p. 255-72.

15. Pazirandeh S, Lo CW, Burns DL. Overview of watersoluble vitamins. In: Rose BD, editor. UpToDate (book on CD-ROM). Wellesley, MA, 2002. 
16. Baron RB. Nutrition. In: Tierney LM, McPhee SJ, Papadakis MA, editors. Current Medical Diagnosis \& Treatment. $43^{\text {th }}$ ed. New York:McGraw-Hill, 2004. p.1203-32.

17. Chang CG, Adams-Huet B, Provost DA. Acute postgastric reduction surgery (APGARS) neuropathy. Obes Surg 2004; 14:182-9.

\section{Endereço para correspondência:}

Lilian Freitas de Assunção Alves

Rua Monte Alegre 851, ap. 202

30240-230 Belo Horizonte, MG

E-mail: lilianfaa@yahoo.com 